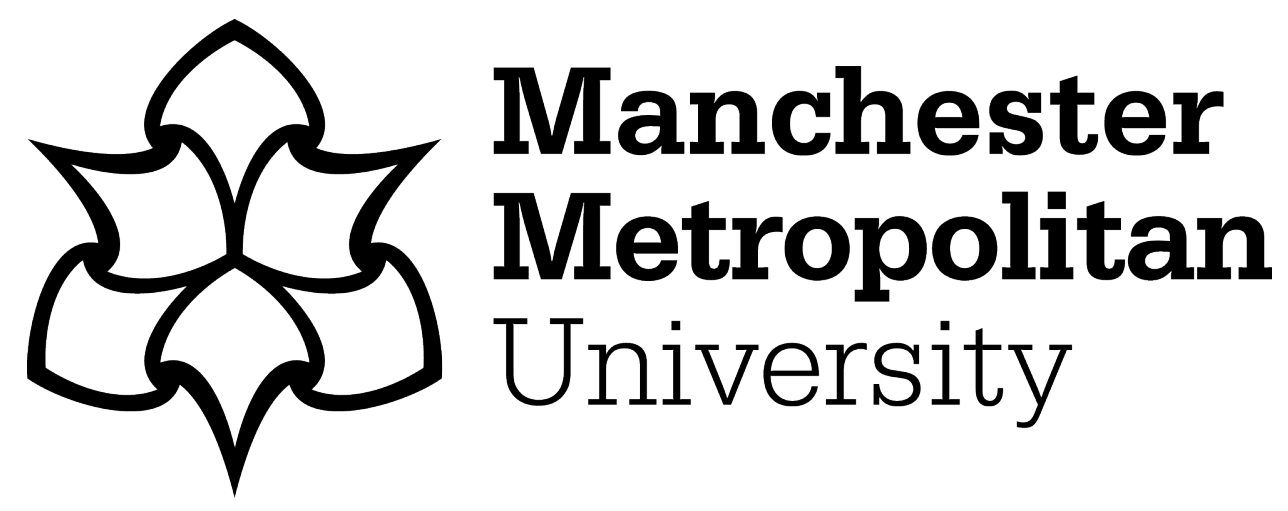

Jayes, Mark ORCID logoORCID: https://orcid.org/0000-0002-0371-7811 and Palmer, Rebecca (2014) Initial evaluation of the Consent Support Tool: A structured procedure to facilitate the inclusion and engagement of people with aphasia in the informed consent process. International Journal of Speech-Language Pathology, 16 (2). pp. 159-168. ISSN 1754-9507

Downloaded from: https: //e-space.mmu.ac.uk/624138/

Version: Accepted Version

Publisher: Taylor \& Francis

DOI: https://doi.org/10.3109/17549507.2013.795999

Please cite the published version 


\title{
Initial evaluation of the Consent Support Tool: A structured procedure to facilitate the inclusion and engagement of people with aphasia in the informed consent process
}

\author{
MARK JAYES ${ }^{1} \&$ REBECCA PALMER ${ }^{2}$ \\ ${ }^{1}$ Sheffield Teaching Hospitals NHS Foundation Trust, Sheffield, UK, and ${ }^{2}$ Sheffield Teaching Hospitals NHS Foundation \\ Trust and University of Sheffield, Sheffield, UK
}

\begin{abstract}
This study evaluated the Consent Support Tool (CST), a procedure developed to identify the optimum format in which to present research information to people with different severities of aphasia, in order to support their understanding during the informed consent process. Participants were a convenience sample of 13 people with aphasia who had mixed comprehension ability. The CST was used to profile each participant's language ability and identify an information format that should maximize her/his understanding. Next, participants were shown information presented in three formats: standard text and two "aphasia-friendly" versions providing different levels of support. Participants' understanding of the information was measured for each format. The format recommended by the CST was compared with the format observed to maximize understanding for each participant. The CST accurately predicted the optimum format for 11/13 participants and differentiated people who could understand fully with support from those who could not in 12/13 cases. All participants interviewed (10/10) found the adapted formats helpful and $9 / 10$ preferred them to the standard version. These findings suggest that the CST could usefully support researchers to determine whether a person with aphasia is likely to be able to provide informed consent, and which information format will maximize that individual's understanding. The CST and different information formats are available as Supplementary Appendices to be found online at http://www.informahealthcare.com/doi/abs/10.3109/ 17549507.2013.795999.
\end{abstract}

Keywords: Aphasia, stroke, case series

\section{Introduction}

People with aphasia, particularly severe aphasia, are often excluded from participating in research studies (Dalemans, Wade, van den Heuvel, \& de Witte, 2009). Even studies specifically designed to investigate the experience of stroke survivors have excluded this group (Townend, Brady, \& McLaughlan, 2007). As a result, the generalizability of research findings relating to the aphasic stroke population will be limited. Furthermore, such research practice denies people with aphasia the right to inhabit the research participant role.

Even when research studies are designed to include people with aphasia, challenges inherent to the process of gaining informed consent from this group mean that such individuals are vulnerable during the recruitment process. Many researchers appear to lack awareness of how communication difficulties might impact on an individual's ability to engage in the informed consent process (Penn, Frankel, Watermeyer, \& Müller, 2009). Impaired communication skills may "mask" an individual's ability to make informed decisions (Ferguson, Duffield, \& Worrall, 2010; Kagan \& Kimelman, 1995). Researchers with limited understanding of aphasia might erroneously conclude that a person with impaired expressive language or speech might lack sufficient ability to provide informed consent (Stein \& Brady Wagner, 2006). In contrast, inaccurate judgement of an individual's comprehension skills might lead researchers to assume incorrectly that the person has intact capacity to consent (Savage, 2006). As a consequence, people with aphasia might be recruited to studies without fully understanding important implications of their participation. This could expose participants unknowingly to potentially harmful and distressing experiences.

Currently, the evidence-base relating to the process of gaining informed consent from people with 
aphasia is extremely limited (Penn et al., 2009). A need has been identified for studies specifically to investigate methods that may facilitate the process (Brady Wagner, 2003; Carling-Rowland \& Wahl, 2010). One potential method is to provide information that has been adapted to meet the individual needs of people with impaired comprehension, in order to maximize their participation within decisionmaking (Braunack-Mayer \& Hersh, 2001; Carlsson, Paterson, Scott-Findlay, Ehnfors, \& Ehrenberg, 2007; White-Bateman, Schumacher, Sacco, \& Appelbaum, 2007). In England and Wales, the provision of accessible information forms part of the legal requirements of the Mental Capacity Act (Office of Public Sector Information, 2005), which states that every effort should be made to support individuals to make an informed decision, and that information should be provided in different formats for those who have difficulty understanding information in traditional written or spoken forms.

Evidence supports the use of generic "aphasiafriendly" principles to make written information more accessible to people with aphasia (Brennan, Worrall, \& McKenna, 2005; Dalemans et al., 2009; Rose, Worrall, \& McKenna, 2003; Rose, Worrall, Hickson, \& Hoffman, 2011). Such principles involve adapting information using modifications to both language and design; these usually include limiting the amount of text presented on each page, simplifying the language used, choosing larger font sizes, and including pictures to illustrate salient information (Connect, 2007; Worrall, Rose, Howe, Brennan, Egan, Oxenham, et al., 2005).

However, as people with aphasia present with different patterns and severities of communication difficulty, it cannot be assumed that adapting information using these conventions will enable all individuals to understand sufficiently for the purposes of providing informed consent. Such approaches have been shown to benefit individuals with mild-tomoderate aphasia (Brennan et al., 2005; Rose et al., 2003). People with more severe aphasia, and particularly those with significant reading difficulties, are unlikely to fully understand information when it is adapted using generic principles. A more helpful and acceptable approach would be to tailor information to the language ability and needs of the individual and to acknowledge that people with more severe language difficulties are less likely to be able to give informed consent, even when information is adapted to provide high levels of support. For such individuals, adapted information formats can play a useful role in increasing their involvement and engagement in the consent process.

The Consent Support Tool (CST) is based on a structured procedure developed by Palmer and Paterson (2011) to facilitate recruitment of people with aphasia to a computer therapy study (Palmer, Enderby, Cooper, Latimer, Julious, Paterson, et al., 2012). The CST is designed to enable researchers to differentiate individuals who are likely to be able to provide informed consent with support from those who are not. It uses individual levels of comprehension ability to identify the optimum format in which to present information to each person, in order to maximize understanding and ensure that consent is likely to be fully informed. The CST, thus, provides a practical tool to support researchers to carry out more ethical recruitment of people with aphasia and also represents a unique attempt to make accessible information more responsive to individual needs.

The CST (shown in the Supplementary Appendix) involves an informal screening test of an individual's ability to understand and use spoken and written language. The CST links this information to potential strategies the researcher might use to support participants to understand research information and express questions and opinions about their participation. The ability to understand words and sentences is tested by asking individuals to identify objects or carry out commands in response to either spoken or written stimuli; the ability to read more than a single sentence is tested by asking individuals to read a short paragraph designed to reflect a decisionmaking scenario and then answer a series of factual questions requiring only "yes" or "no" responses.

The results of this screen are used to place the individual within one of four language profiles, based on how much spoken and written information $s / h e$ is able to understand (as proposed by Palmer and Paterson, 2011). For each language profile, the CST proposes an information format that should facilitate optimal understanding: (1) if an individual is able to read written paragraphs, research information should be written using lay terminology with important concepts placed in bold; (2) if $s /$ he is able to read three key words (words that convey salient information) in a sentence, information should be formatted using the aphasia-friendly recommendations proposed by Connect (2007); (3) if s/he is able to understand two key words in written and spoken sentences, information should be presented using the same aphasia-friendly formatting and delivered one concept at a time on Microsoft Office PowerPoint slides; the researcher should explain each concept using "total communication" strategies (e.g., using speech, writing, drawing, gesture, mime); and (4) if an individual understands less than two key written or spoken words in a sentence, the CST proposes that $\mathrm{s} /$ he will be unlikely to understand sufficiently for the purposes of providing informed consent and recommends that relative or carer assent should be taken instead.

The primary aim of this study was to carry out an initial evaluation of the CST's validity, by investigating its accuracy in predicting the optimum style of information to use to support people with different severities of comprehension impairment 
to understand research information. The second aim was to examine whether providing increased levels of support to understand information was associated with increased levels of comprehension. The third aim was to explore participants' views on the different information formats used, in order to measure their acceptability to people with aphasia and identify ways in which they could be refined.

\section{Method}

The study used a case series, mixed methods design. A sample of people with aphasia was recruited to take part in a quantitative evaluation of the accuracy of the CST in predicting optimum information style to facilitate informed consent. To complement this, short semi-structured interviews were conducted with the same participants in order to collect qualitative data about their views on the adapted information styles used. This research was approved by the University of Sheffield School of Health and Related Research Ethics Committee.

\section{Participants}

Participants were eligible for inclusion in this study if they had a diagnosis of aphasia. Individuals were not eligible if they presented with severe visual or cognitive difficulties which would limit their ability to process visual information presented within the experimental procedure. All participants of an ongoing computer aphasia therapy study were approached (Palmer et al., 2012). Due to the relatively small number of participants available (24), a convenience sampling strategy was used, whereby all individuals expressing an interest in participating in the study were recruited. Participants were categorized as having a mild, moderate, or severe comprehension impairment on the basis of their scores on the language comprehension sub-tests of the Comprehensive
Aphasia Test (CAT) (Swinburn, Porter, \& Howard, 2005); these data were collected previously (Palmer et al., 2012).

Consent or assent to take part in the study was obtained for 14 people. One participant (002) withdrew from the study during the experimental session. The remaining sample was composed of nine male and four female participants, with ages ranging from 38-82 years (mean age 61.92 years). Participants had all experienced at least one CVA between 3-13 years prior to testing (mean time 6.23 years). The sample included participants with different types of aphasia. Participant characteristics are shown in Table I.

\section{Data collection and materials}

Accuracy of CST in predicting optimum information style. CST accuracy was measured by comparing the information style predicted to be optimal for each participant with the version of a mock participant information sheet (PIS) that enabled the participant to achieve maximum understanding of study-related information. Three versions of a mock participant information sheet were created, corresponding to the information formats proposed by the CST: PIS 1 presented standard written information using lay terminology and key concepts placed in bold; PIS 2 presented information formatted using the standard aphasia-friendly principles proposed by Connect (2007), including non-copyrighted pictures selected from the Microsoft Office 2007 ClipArt and Google Images databases; PIS 3 presented individual concepts on Microsoft Office 2007 PowerPoint slides using aphasia-friendly formatting. The information contained within each PIS version described the same fictitious study involving a physical exercise therapy unrelated to speech-language pathology.

A picture card sorting task was used to measure each participant's understanding of the information contained within the three PIS versions. This task

Table I. Participant characteristics $(n=14)$.

\begin{tabular}{|c|c|c|c|c|c|}
\hline $\begin{array}{l}\text { Participant } \\
\text { identification } \\
\text { number }\end{array}$ & $\begin{array}{c}\text { Age } \\
\text { (years) }\end{array}$ & Gender & $\begin{array}{l}\text { Type of } \\
\text { aphasia }\end{array}$ & $\begin{array}{c}\text { Severity of } \\
\text { comprehension } \\
\text { impairment }\end{array}$ & $\begin{array}{c}\text { Time since } \\
\text { CVA }\end{array}$ \\
\hline 001 & 55 & Male & Non-fluent & Mild & 6 years \\
\hline 002 & 65 & Male & Non-fluent & Mild & 10 years \\
\hline 003 & 53 & Male & Non-fluent & Mild & 10 years \\
\hline 004 & 67 & Male & Non-fluent & Mild & 7 years \\
\hline 005 & 68 & Male & Non-fluent & Mild & 4 years \\
\hline 006 & 78 & Male & Non-fluent & Severe & 3 years \\
\hline 007 & 65 & Male & Global & Severe & 8 years \\
\hline 008 & 52 & Male & Non-fluent & Severe & 4 years \\
\hline 009 & 38 & Female & Global & Severe & 5 years \\
\hline 010 & 55 & Female & Non-fluent & Moderate & 13 years \\
\hline 011 & 68 & Male & Global & Severe & 6 years \\
\hline 012 & 82 & Female & Fluent & Moderate & 7 years \\
\hline 013 & 50 & Male & Fluent & Moderate & 5 years \\
\hline 014 & 74 & Female & Non-Fluent & Moderate & 3 years \\
\hline
\end{tabular}


required the participant to sort pictures corresponding to important concepts contained within the information in each PIS from pictures corresponding to concepts not contained within it. Participants were considered to have understood the key concepts fully if they were able to sort the pictures with $100 \%$ accuracy. Picture sorting was used because this paradigm provides a useful means of assessing knowledge and comprehension of information in situations when individuals have difficulty understanding information expressed linguistically and/or have difficulty providing verbal responses.

Twenty picture cards corresponding to important categories of concepts contained within the information about the fictitious study were created. These pictures were different to those used in the participant information sheets to reduce the likelihood of participants completing the task using a picture matching strategy rather than on the basis of their understanding of concepts. The concept categories tested related to the general topic of the research, the research setting, the use of randomized participation allocation, the specific tasks required of participants during the study, the voluntary nature of participation and the right to withdraw, and also participant confidentiality. These categories are consistent with the types of information proposed within the literature as essential to the process of gaining informed consent to participation in research (e.g., Penn et al., 2009; Savage, 2006).

In order to reduce the likelihood that any observed improvements in comprehension were due to learning effects secondary to the repeated presentation of the same information across different PIS versions, information was presented in descending order of comprehension difficulty (PIS1 $\rightarrow$ PIS2 $\rightarrow$ PIS3). In order to verify whether improvements in understanding might still be due to learning effects, the cumulative effect of reading the same information twice was measured. This was achieved by asking participants to read PIS 1 twice and comparing scores on the picture sorting task for each reading.

Participants' views on adapted information formats. An interview guide was designed to generate specific data on a limited number of salient topics, in order to ensure brevity (Carlsson et al., 2007). Topics were identified from a review of the literature on accessible information. The topics related primarily to which aspects of the adapted information each participant preferred and found most helpful. Questions were designed to be short and involve simple language and high frequency words (Dalemans et al., 2009). Pictures and visual rating scales were produced to facilitate participants' understanding of questions and concepts during the interview, in order to maximize the validity of their responses (Luck \& Rose, 2007). These materials were also designed to enable participants to express their opinions non-verbally if necessary (e.g., by pointing at a picture or rating scale).

\section{Procedure}

Informed consent to participate was obtained using the current standard method employed in studies recruiting people with communication difficulties (e.g., Penn et al., 2009). The first author (a speech-language pathologist) provided aphasiafriendly information about the study and discussed this with each participant before asking closed questions to establish if the individual understood what participation would involve. When the author was satisfied that a participant appeared to understand, written consent was taken using a consent form containing information with aphasia-friendly formatting. When a participant appeared to have difficulty understanding, but seemed satisfied with the general idea of participating, assent was taken from a relative.

Experimental sessions were carried out in participants' own homes and lasted between 1-2 hours. The author administered the CST with each participant. Next, each participant was invited to read PIS1. The author then presented 20 picture cards and asked the participant to sort these into three piles: one corresponded to concepts contained within the information, a second corresponded to concepts not contained within the information, and a third was to be used when participants did not know whether or not the concepts were contained within the information. Participants were encouraged to refer back to the PIS if they needed to remind themselves of particular information in order to complete the task.

If the participant was unable to achieve $100 \%$ accuracy on the sorting task, s/he was invited to reread PIS 1 and complete the sorting task in exactly the same way as before. If the participant was still unable to achieve $100 \%$ accuracy, s/he was invited to read PIS 2 and complete the sorting task again. If the participant achieved less than $100 \%$, s/he was invited to look at the PIS 3 PowerPoint slides presented on a laptop computer; during this presentation, the author explained each concept and checked participants' understanding of each using total communication methods. The picture sorting task was then repeated for the final time.

Next, each participant was invited to take part in the short interview. The author adjusted the content and structure of each interview, as well as the language and communication strategies used, to meet the needs of each individual. Not all individuals were able to participate fully in the interviews, due to the severity of their comprehension and/or expressive language difficulties. The interviews were recorded with consent for later transcription; the author also made written notes of non-verbal behaviours (e.g., facial expressions, pointing responses to visual scales). Interviews lasted between 5-10 minutes, depending on the responses of individual participants. 


\section{Data analysis}

Quantitative data. The information format predicted for each participant using the CST was compared against the PIS version actually observed to support the participant to understand all the information. A Cohen's kappa statistic (Cohen, 1960) was calculated to summarize the level of agreement between these data. This statistic provides a measure of agreement that is adjusted to account for the amount of agreement that might be expected by chance (Bland, 2008). Non-parametric statistical tests were used to investigate differences in picture sorting task scores across each PIS version. Statistical analysis was carried out using IBM SPSS 19 software.

Qualitative data. The author transcribed each interview verbatim. Information about non-verbal communication behaviours that contributed to the meaning of individual responses was also recorded, in order to increase the validity of the data (Luck \& Rose, 2007). QSR NVivo 8 software was used to facilitate thematic analysis using a Framework approach (Ritchie \& Spencer, 1994). Whenever the meaning of a participant's response appeared ambiguous, the response was excluded from analysis, in order to maintain the validity and reliability of the data (Luck \& Rose, 2007). Content analysis was used to quantify which PIS versions participants preferred and found most helpful. Major themes arising during data analysis were summarized and illustrated with sections of original data.

\section{Results}

Accuracy of CST in predicting the optimum style of information to support comprehension

Table II displays individual participant scores on the picture sorting task for each version of the participant information sheet tested, the PIS version predicted by the CST to support each participant's understanding optimally, and the version observed experimentally to enable the participant to understand all the information. Note PIS 4 refers to the situation where participants were unable to sort all the pictures accurately and it was concluded that they would be unlikely to be able to provide informed consent themselves.

Table II shows that no participant was able to complete the picture sorting task with complete accuracy after reading PIS 1 (standard text). Three participants with mild comprehension impairments (003, 004, 005) and one participant with moderately impaired comprehension (014) achieved $100 \%$ accuracy after reading one of the adapted information versions. Most participants (9/13) were still unable to understand the information fully after reading PIS 3.

The optimum PIS version predicted by the CST matched the version observed to facilitate optimum comprehension for 11 of the 13 participants. The Cohen's kappa statistic for the level of agreement between these two data sets was calculated as .708, which suggests there is "substantial" agreement between the type of information the CST predicted people would need and the type they actually did need to facilitate maximum understanding (Landis \& Koch, 1977). The $95 \%$ confidence interval for the Cohen's kappa statistic was .257-1.000.

The CST did not accurately identify the optimum style of information to provide to participants 001 and 005. Participant 001 had difficulty understanding a number of concepts within each PIS version and often appeared to rely on heuristic knowledge to help him complete the picture sorting task. It appears that the CST may have over-estimated this participant's ability to understand both spoken and written language. Participant 005 required the higher level of support provided by PIS 3 to

Table II. Picture sorting task scores and predicted and observed optimum participant information sheet (PIS) version for each participant.

\begin{tabular}{|c|c|c|c|c|c|c|}
\hline \multirow{2}{*}{$\begin{array}{l}\text { Participant } \\
\text { identification } \\
\text { number }\end{array}$} & \multicolumn{4}{|c|}{$\begin{array}{l}\text { Picture sorting task score } \\
(\text { minimum score }=0, \text { maximum score }=20)\end{array}$} & \multirow[b]{2}{*}{$\begin{array}{c}\text { Predicted } \\
\text { optimum PIS }\end{array}$} & \multirow{2}{*}{$\begin{array}{l}\text { Observed optimum PIS } \\
\text { (enabling participant to } \\
\text { achieve } 20 / 20 \text { ) }\end{array}$} \\
\hline & PIS 1 ( $1^{\text {st }}$ reading) & $\begin{array}{c}\text { PIS } 1 \\
\left(2^{\text {nd }} \text { reading }\right)\end{array}$ & PIS 2 & PIS 3 & & \\
\hline 001 & 16 & 15 & 19 & 19 & 2 & 4 \\
\hline 003 & 17 & 17 & 20 & NT & 2 & 2 \\
\hline 004 & 19 & 18 & 20 & NT & 2 & 2 \\
\hline 005 & 17 & 17 & 18 & 20 & 1 & 3 \\
\hline 006 & NA & NA & NA & 11 & 4 & 4 \\
\hline 007 & 8 & NA & 14 & 12 & 4 & 4 \\
\hline 008 & 14 & 16 & 15 & 19 & 4 & 4 \\
\hline 009 & 8 & NA & 13 & 17 & 4 & 4 \\
\hline 010 & 8 & NA & 10 & 13 & 4 & 4 \\
\hline 011 & NA & NA & 12 & 11 & 4 & 4 \\
\hline 012 & $\mathrm{D}$ & NA & $\mathrm{D}$ & $\mathrm{D}$ & 4 & 4 \\
\hline 013 & NA & NA & NA & 15 & 4 & 4 \\
\hline 014 & 12 & 10 & 15 & 20 & 3 & 3 \\
\hline
\end{tabular}

NT: Not tested by author as participant had achieved maximum score on previous PIS; NA:Task not attempted by participant; D: Task discontinued by author as participant did not appear to understand task procedure. 
complete the picture sorting task accurately. Two factors might explain this disparity. First, intermittent loud environmental noise during the experimental session may have affected his ability to attend to the information. Secondly, this participant appeared anxious to perform well during the experimental session, which may have adversely affected his performance.

\section{Changes in knowledge gained across different information formats}

Participants' picture sorting scores were found to differ significantly across the different PIS versions $\left(\chi^{2}(2)=15.081, p=.001\right)$. Post-hoc analysis found that scores were significantly higher after reading PIS 2 than after reading PIS $1(Z=-2.527, p=$ .012). Similarly, scores were significantly higher after reading PIS 3 compared with after reading PIS $1(Z=-2.809, p=.005)$. However, scores were not significantly higher after reading PIS 3 compared with after reading PIS $2(\mathrm{Z}=-2.255, \mathrm{p}=.024)$. These results suggest that participants were able to understand more information when they were given aphasia-friendly information, but that increased levels of support or adaptation were not associated with significantly increased levels of comprehension. There was no significant difference between scores for each reading of PIS 1 ( $Z=-1.000$, $\mathrm{p}=.317)$. This suggests that any observed increases in participants' picture sorting scores for the different PIS versions were more likely to be due to increased levels of understanding rather than a learning effect.

\section{Participants' views on adapted information formats}

Complete interview data were obtained for $10 / 13$ participants. All 10 respondents stated that they found one of the adapted formats (PIS 2 or PIS 3) the most helpful type of information. Nine out of 10 participants preferred one of the adapted formats to the standard information format in PIS 1. The exception to this was participant 001 .

\section{Use of pictures}

Nine out of 10 respondents said that they liked the use of pictures to supplement the text in the adapted versions. Participant 003 reported that the inclusion of pictures helped to simplify the information and provided a means for him to limit the amount of information to read. He also suggested that pictures helped him to feel orientated within the text and remember parts of the information:

The pictures help you remember yeah...it's like reading a book (points to PIS 1) it's harder for you to go back and there isn't no picture.
Participant 005 reported that he found the pictures engaged his attention and helped him to remember aspects of the information. Participant 001 was the only respondent who reported that he did not like the use of pictures; he suggested that he found the pictures childish and that he felt insulted by them.

\section{Amount of written information presented}

Eight participants reported that they preferred the adapted information because of the use of different methods to limit the amount of information presented. For example, participants 013 and 014 appreciated the staged presentation of individual concepts in PIS 3. Participant 005 preferred PIS 2 to PIS 1 because the standard PIS version contained a lot of information:

Yes exactly ... by the time you get down here (points halfway down page two of PIS 1) you think crikey ... I'd better go back and see (points to page 1) you know it does get a little bit wearing after all those words you know.

This participant also suggested that the use of bullet points to break up information and short sentences were beneficial.

\section{Use of total communication strategies}

Five participants stated that they found the author's use of total communication strategies to explain the information in PIS 3 helpful. Participant 014 explained that she would find it easier to understand information if she were able to talk to someone about it:

Yes yes ... because sometimes it's just not there (points to head) as well so of course ... with someone there you can talk to me you see so that's a lot better.

\section{Participants' suggestions for improving the adapted information further}

Two participants observed that the font used for the PowerPoint presentation of PIS 3 (size 32) was larger than that used for PIS 1 and PIS 2 (size 14). They suggested that PIS 2 could be improved by incorporating larger font. When commenting on PIS 3, participant 013 stated:

\footnotetext{
... because it's larger it's great ... if it's if it's this sort of size (points to PIS 1) it's hard for you to suss out ... but when it's the computer's larger and the sentences not large it's easier for you to suss out.
}

One participant (009) stated that she believed the information contained within all of the PIS versions would be difficult to understand even for people who did not have aphasia. Another participant (010) commented that, even in the adapted information 
versions, some of the language was too complex and included certain words that were too long.

\section{Discussion}

Accuracy of CST in predicting an optimum information format for people with aphasia

The primary aim of this study was to investigate whether the CST could be used to accurately predict the most appropriate information format to use to support an individual with aphasia to understand sufficient information about a research study in order to provide informed consent. The results demonstrate that the CST accurately identified the optimum information style for 11 of the 13 participants. Statistically, this level of agreement is substantial. The large confidence interval for the kappa statistic, however, suggests that our estimate for the statistic is imprecise and that the true value could be as high as 1.000 , indicating "near perfect" agreement, or as low as .257, which would only represent "fair" agreement according to the Landis and Koch (1977) framework. The probable cause for this lack of precision is the small sample size used.

Although the sample included almost equal numbers of participants with different severities of comprehension impairment, the majority of participants were unable to complete the picture sorting task with complete accuracy after reading any of the different versions of participant information. This suggests that, even with the aid of adapted information formats, some people with aphasia may be unlikely to be able to understand sufficient information for the purposes of making informed decisions. The CST accurately differentiated individuals who were able to understand the information fully using the adapted information formats from those who were not in $12 / 13$ cases. This indicates that the CST may provide a valuable means for researchers to rapidly identify those participants who are likely to be able to provide informed consent with an appropriate level of support and those for whom assent may need to be taken from an advocate.

As one might expect, the results indicate that the adapted information formats were most beneficial to those participants with milder comprehension deficits. This finding is consistent with previous studies involving aphasia-friendly information (Brennan et al., 2005; Rose et al., 2003). Although comparing results across studies is problematic, it does appear that the adaptation of information using aphasiafriendly principles is likely to benefit some but not all people with aphasia, and that those with moderateto-severe aphasia are less likely to be able to understand such information sufficiently for the purposes of providing informed consent. These observations support the rationale for the CST to differentiate individuals who are likely to be able to provide informed consent with support from those who are not and to identify what style of information will support them to be able to do so.

Although adapted information cannot support all people with aphasia to provide informed consent, the fact that adapted formats might enable them to understand more information means that such formats can still assist an individual to have some involvement in decisions around participation in research. It is, therefore, important to understand the relative usefulness of the different information formats suggested by the CST.

Overall, participants understood more information with the adapted formats in PIS 2 and PIS 3 compared with the standard format PIS 1. This improvement in performance appeared to be related to the use of different information styles and not merely due to participants learning the material over successive readings. The total communication approach and staged delivery of information in PIS 3 were designed to provide additional support to the aphasia-friendly information format. However, as a group, participants did not achieve significantly higher scores on the comprehension task after reading PIS 3 compared with when they read PIS 2 . This suggests that PIS 3 did not provide additional benefits to participants' understanding, which might lead one to conclude that PIS 3 was superfluous. However, the majority of participants who were shown PIS 3 stated in interview that they preferred this style and delivery of information and found it the most helpful version. This suggests that PIS 3 did provide valuable support to participants. It is recommended, therefore, that all PIS versions should be maintained within the CST.

\section{Acceptability of adapted information formats}

The qualitative data collected in this study support the use of aphasia-friendly design features and total communication strategies as acceptable ways to assist people with aphasia to maximize their understanding of research information. However, it should be recognized that some individuals may not appreciate the use of modified information, and particularly the inclusion of pictures, which they may find insulting. These mixed views on adapted information formats are consistent with previous studies involving people with aphasia (Dalemans et al., 2009; Eames, McKenna, Worrall, \& Read, 2003; Kagan \& Kimelman, 1995; Rose et al., 2003; Rose, Worrall, Hickson, \& Hoffmann, 2012). Where possible, people with aphasia should be offered a choice of information formats and also should be involved in the design of research information materials.

\section{Limitations of current study}

This was an observational study with a case series design, which means it was vulnerable to bias. The 
small sample size used is likely to have limited its representativeness and thus compromised both the generalizability of the results and the precision of the estimated statistical level of agreement. Blinded data collection and analysis were not possible, which may also have introduced bias. Although respondent validation techniques were used to verify the meanings of verbal and non-verbal responses made by participants during the interviews, the processes of recording, transcribing, and analysing data all involved subjective decisions.

Due to time restrictions, most participants were recruited, consented, and tested in one session. It is possible therefore that fatigue affected participants' ability to understand and express language. This is particularly pertinent for participants with the most severe language difficulties, who were more likely to be shown all PIS versions and, thus, require a longer, potentially more tiring session. Finally, it could also be argued that performance on the picture sorting task was more indicative of participants' ability to recall information rather than understand it.

\section{Role of the CST within the informed consent process}

The informed consent process requires researchers to assess whether an individual has the mental capacity to make an informed decision about participating in a research study. This assessment involves establishing whether the individual is able to understand, retain, and weigh up information relating to participation in a research study and then communicate her/his decision about whether or not to participate (Mental Capacity Act, 2005). It is important to recognize the role of the CST within this process: the tool does not replace the assessment of capacity and cannot provide definitive evidence of an individual's ability to make an informed decision. Rather, the CST is designed to support researchers to provide appropriate accessible information to people with aphasia, in order to help them to understand more information, and it provides an indication of whether or not an individual with aphasia is likely to be able to understand sufficiently for the purposes of providing informed consent; it also suggests strategies to use to support an individual with aphasia to ask questions, express their concerns, and communicate a decision.

After using the CST to indicate the most appropriate way to present information to the individual, the researcher then needs to check the information has been understood and weighed up by that individual, before supporting her/him to express a decision about participation. Different methods can be used to achieve this. Ideally, potential participants should be asked to describe a study in their own words and invited to ask questions about it and discuss what participation might involve. In addition to demonstrating how much people understand about participating in a study, such discussions can serve to increase their understanding of study information and also their engagement within the consenting process. Individuals with receptive and/or expressive aphasia can be enabled to participate in these discussions through the use of total communication strategies (specific strategies are suggested within the CST). For people with more severe expressive difficulties, Stein et al. (2006) propose a useful model of facilitated consent, whereby an advocate for the person with aphasia who knows her/him well can ask questions on her/his behalf. Researchers can verify that people with aphasia understand specific aspects of study information by asking them closed questions, including those requiring only "yes/no" answers, or by asking them to choose between forced alternatives. Individuals with more severe receptive aphasia might be asked to demonstrate their understanding by sorting pictures according to their relevance to the study (as participants were asked to do in this study), or according to the sequence in which events would occur in the study.

\section{Implications of this study for clinical practice and future research}

(1) The CST can support researchers to determine whether or not a person with aphasia is likely to be able to provide informed consent independently, and which type of information format will maximize that individual's understanding of research information.

(2) People with milder rather than moderate or severe comprehension difficulties are more likely to be able to use adapted information formats to understand research information.

(3) The provision of adapted, aphasia-friendly information can increase understanding of research information and thereby facilitate the engagement of people with aphasia within the consenting process, even in situations when assent to participate needs to be taken from an advocate.

(4) People with aphasia have individual preferences regarding information provision; where possible, research information should be made available in a choice of formats.

(5) In addition to providing paper and/or computer-based information, people with aphasia should be offered opportunities to discuss the nature of their participation in a research study using a range of facilitative communication strategies, in order to maximize their understanding and engagement during the consenting process.

(6) Further, more thorough evaluation of the CST is feasible and warranted. The limitations described above suggest ways in which the design and methodology could be 
improved in order to increase the validity and reliability of data.

(7) Future investigations should evaluate how easily and effectively the CST can be used by researchers without specialist knowledge and skills in working with people with aphasia. It will be important to ascertain if researchers consider the CST to be a useful tool. The authors acknowledge that use of the CST and the development of accessible information materials place additional demands on researchers' time and resources. However, the CST is designed to be administered rapidly and the accessible formats it proposes can be prepared relatively easily and quickly in advance of the recruitment phase of a research project using a range of freely available online resources (as described earlier).

(8) The scope of the current evaluation could be extended to investigate use of the CST with different clinical populations (e.g., people with aphasia at different stages of recovery poststroke or people with cognitive-communication difficulties), or as a means to support people to make informed decisions and give consent in a range of contexts beyond participation in research (e.g., decisions around medical treatments, living arrangements, or financial management).

\section{Conclusion}

This evaluation suggests that the Consent Support Tool has the potential to facilitate the process of obtaining informed consent from people with aphasia, by supporting researchers to provide more accessible information formats that are both acceptable to people with aphasia and more responsive to their individual needs. This should enable researchers to engage in more ethical recruitment processes and facilitate the wider inclusion and participation of people with aphasia within research. Beyond this context, the CST also has the potential to be used with different clinical populations who present with communication difficulties and by different professionals within different settings, for purposes other than recruitment to research studies.

\section{Acknowledgements}

Mr Jayes undertook this research whilst in receipt of a National Institute for Health Research (NIHR) Clinical Academic Training (CAT) Masters in Clinical Research studentship award. The study was supervised by Dr Rebecca Palmer and was supported by the NIHR Collaboration for Leadership in Applied Health Research and Care for South Yorkshire (CLAHRC SY). CLAHRC SY acknowledges funding from the National Institute for Health Research.
The views and opinions expressed are those of the authors, and not necessarily those of the NHS, the NIHR or the Department of Health. CLAHRC SY would also like to acknowledge the participation and resources of their partner organizations. Further details can be found at www.clahrc-sy.nihr.ac.uk.

Declaration of Interest: The authors report no conflicts of interest. The authors alone are responsible for the content and writing of the paper.

\section{References}

Bland, M. (2008). Measuring health and disease. Cohen's kappa. Percentage agreement: A misleading approach. York: University of York.http://www-users.york.ac.uk/ mb55/msc/clinimet/week4/ kappash2.pdf.

Brady Wagner, L. C. (2003). Clinical ethics in the context of language and cognitive impairment: Rights and protections. Seminars in Speech and Language, 24, 275-284.

Braunack-Mayer, A., \& Hersh, D. (2001). An ethical voice in the silence of aphasia: Judging understanding and consent in people with aphasia. The fournal of Clinical Ethics, 12, 388-396.

Brennan, A., Worrall, L., \& McKenna, K. (2005). The relationship between specific features of aphasia-friendly written material and comprehension of written material for people with aphasia: An exploratory study. Aphasiology, 19, 693-711.

Carling-Rowland, A., \& Wahl, J. (2010). The evaluation of capacity to make admission decisions: Is it a fair process for individuals with communication barriers? Medical Law International, 10, 171-190.

Carlsson, E., Paterson, B. L., Scott-Findlay, S., Ehnfors, M., \& Ehrenberg, A. (2007). Methodological issues in interviews involving people with communication impairments after acquired brain damage. Qualitative Health Research, 17, 1361-1371.

Cohen, J. (1960). A coefficient of agreement for nominal scales. Educational and Psychological Measurement, 20, 37-46.

Connect. (2007). Including people with communication disability in stroke research and consultation: $A$ guide for researchers and service providers. London: Connect Communication Disability Network.

Dalemans, R., Wade, D. T., van den Heuvel, W. J. A., \& de Witte, L. P. (2009). Facilitating the participation of people with aphasia in research: A description of strategies. Clinical Rehabilitation, 23, 948-959.

Eames, S., McKenna, K., Worrall, L., \& Read, S. (2003). The suitability of written education materials for stroke survivors and their carers. Topics in Stroke Rehabilitation, 10, 70-83.

Ferguson, A., Duffield, G., \& Worrall, L. (2010). Legal decisionmaking by people with aphasia: Critical incidents for speech pathologists. International fournal of Language and Communication Disorders, 45, 244-258.

Kagan, A., \& Kimelman, M. (1995). Informed consent in aphasia research: Myth or reality? Clinical Aphasiology, 23, 65-75.

Landis, J. R., \& Koch, G. G. (1977). The measurement of observer agreement for categorical data. Biometrics, 33, 159-174.

Luck, A. M., \& Rose, M. L. (2007). Interviewing people with aphasia: Insights into method adjustments from a pilot study. Aphasiology, 21, 208-224.

Office of Public Sector Information. (2005). Mental Capacity Act 2005. London: OPSI.

Palmer, R., \& Paterson, G. (2011). One size does not fit all: Obtaining informed consent from people with aphasia. Advances in Clinical Neurosciences and Rehabilitation, 11, 30-31.

Palmer, R., Enderby, P., Cooper, C., Latimer, N., Julious, S., Paterson, G., et al. (2012). Computer therapy compared with 
usual care for people with long-standing aphasia post-stroke: A pilot randomized controlled trial. Stroke, 43, 1904-1911.

Penn, C., Frankel, T., Watermeyer, J., \& Müller, M. (2009). Informed consent and aphasia: Evidence of pitfalls in the process. Aphasiology, 23, 3-32.

Ritchie, J., \& Spencer, L. (1994). Qualitative data analysis for applied policy research. In A. Bryman, \& A. Burgess (Eds.), Analysing qualitative data (pp. 173-194). London: Routledge.

Rose, T., Worrall, L., \& McKenna, K. (2003). The effectiveness of aphasia-friendly principles for printed health education materials for people with aphasia following stroke. Aphasiology, 17, 947-963.

Rose, T. A., Worrall, L. E., Hickson, L. M., \& Hoffmann, T. C. (2011). Aphasia friendly written health information: Content and design characteristics. International fournal of SpeechLanguage Pathology, 13, 335-347.

Rose, T. A., Worrall, L. E., Hickson, L. M., \& Hoffmann, T. C. (2012). Guiding principles for printed education materials: Design preferences of people with aphasia. International Fournal of Speech-Language Pathology, 14, 11-23.

\section{Supplementary material available online}

Supplementary Appendices to be found online at http://www.informahealthcare.com/doi/abs/10.3109/ 17549507.2013.795999
Savage, T. A. (2006). Ethical issues in research with patients who have experienced stroke, Topics in Stroke Rehabilitation, 13, $1-10$.

Stein, J., \& Brady Wagner, L. C. (2006). Is informed consent a "yes or no" response? Enhancing the shared decision-making process for persons with aphasia. Topics in Stroke Rehabilitation, $13,42-46$.

Swinburn, K., Porter, G., \& Howard, D. (2005). The Comprehensive Aphasia Test. Hove, UK: Psychology Press.

Townend, E., Brady, M., \& McLaughlan, K. (2007). Exclusion and inclusion criteria for people with aphasia in studies of depression after stroke: A systematic review and future recommendations. Neuroepidemiology, 29, 1-17.

White-Bateman, S. R., Schumacher, H. C., Sacco, R. L., \& Appelbaum, P. S. (2007). Consent for intravenous thrombolysis in acute stroke: Review and future directions. Archives of Neurology, 64, 785-792.

Worrall, L., Rose, T., Howe, T., Brennan, A., Egan, J., Oxenham, D., et al. (2005). Access to written information for people with aphasia. Aphasiology, 19, 923-929. 